PHYSICAL REVIEW D 74, 019902(E) (2006)

\title{
Erratum: Improved bounds on universal extra dimensions and consequences for Kaluza-Klein dark matter [Phys. Rev. D 73, 095002 (2006).]
}

\author{
Thomas Flacke, Dan Hooper, and John March-Russell
}

(Received 19 June 2006; published 28 July 2006)

DOI: 10.1103/PhysRevD.74.019902

PACS numbers: 12.60.-i, 11.25.Mj, 12.15.Lk, 95.35.+d, 99.10.Cd

In combining our calculation of the Kaluza-Klein (KK) contribution to the $\hat{S}, \hat{T}, \hat{U}, W, X, Y$ observables with the LEPII constraints and SM contributions as discussed by Barbieri, et al. [1] we were unaware that Ref. [1] uses an opposite definition of the sign of all self-energies $\Pi_{X Y}$ compared to the standard convention. In consequence, our KK contributions to the EWPO are wrong by this sign. The corrected contributions to the $\hat{T}$ parameter are shown in Fig. 1 (left).

Our relative sign error between the SM contributions given in [1] and the KK-contributions to the EWPO has a major impact on the fit to the measured values for the EWPO. The corrected fit is given in Fig. 1 (right). As a consequence, the full analysis of the EWPO contributions including the 2-loop SM corrections do not yield a substantial improvement of the constraints on the compactification radius. For a heavy Higgs $m_{H} \sim 800 \mathrm{GeV}$, an inverse compactification radius of $300 \mathrm{GeV}$ is in accord with our corrected analysis. Our corrected results are in agreement with the recent analysis of Gogoladze and Macesanu [2] (for a top mass of $178 \mathrm{GeV}$, the value assumed in Ref. [1]).

The discussion of the prospects of direct and indirect detection of KK dark matter and its' dependence on the compactification radius in Section $\mathrm{V}$ remains fully correct. The analysis of LKP dark matter in the heavy $(1 / R \geq$ $500 \mathrm{GeV}$ ) $\mathrm{KK}$ limit is directly relevant if either the Higgs is found to be light or direct bounds on $1 / R$ from the Tevatron or LHC exceed $\sim 500 \mathrm{GeV}$.

We are very grateful to Ilia Gogoladze and Cosmin Macesanu for discussions of their analysis [2], and we thank Carlos Wagner and the authors of Ref. [2] for follow-up communications which helped us to identify our error.
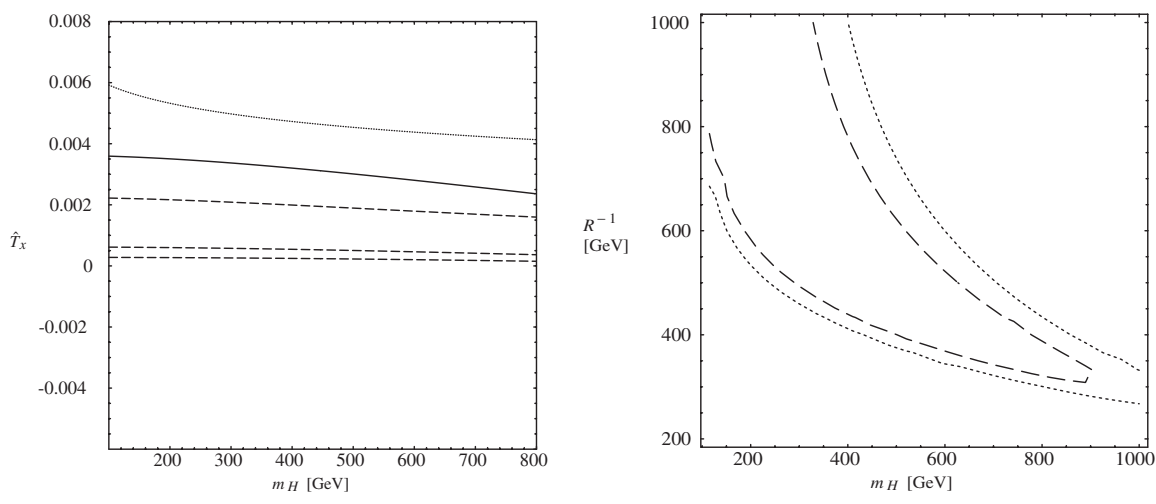

FIG. 1. Correction of Fig. 2 (left) and Fig. 3 (right) of the original paper. (a) The contribution to $\hat{T}$ from the first three KK levels (dashed lines) for $M_{c}=400 \mathrm{GeV}$ as a function of Higgs mass, as well as the sum over the first $10 \mathrm{KK}$ modes (solid line) and the numerically-interpolated Higgs- dependent correction (dotted line) $\epsilon_{1, S M}$. (b) The $95 \%$ (dashed line) and $99 \%$ (dotted line) confidence limit exclusion zones for the UED model, as a function of Higgs mass in the range $115 \mathrm{GeV}$ to $1 \mathrm{TeV}$, and mass $M_{1}=1 / R$ of the lightest $\mathrm{KK}$ excitation in the range $200 \mathrm{GeV}$ to $1 \mathrm{TeV}$.

[1] R. Barbieri, A. Pomarol, R. Rattazzi, and A. Strumia, Nucl. Phys. B703, 127 (2004).

[2] I. Gogoladze and C. Macesanu, hep-ph/0605207. 